nervous system, is not adhered to. Instead the autonomic nervous system is defined as regulating processes not under voluntary or volitional control. This is to adopt a physiological, perhaps a psychological criterion, and would have justified a more adequate discussion of the functional aspects of the system, without which, much of the anatomy has little significance to the clinician. The author also seems to be on somewhat insecure ground in his extension of the term ' autonomic' to include a wide variety of afferent neurones. There is surely nothing ' irrational ' or 'whimsically inconsistent,' to quote his own words, in limiting the term autonomic to an efferent system precisely defined in structural terms. To do so is not to deny the existence of numerous afferent fibres from viscera, or of afferent components in such nerves as the vagus or the splanchnic branches of the sympathetic. It may be that afferent fibres from viscera are less widely recognized than they should be, but it is possible to emphasize their presence without extending the use of the term autonomic so that it loses any precise or definable meaning.

Another result of this very broad interpretation of the meaning of terms, is the inclusion of several topics which to many readers will seem irrelevant to the main purpose of the book. The descriptions given, for example, of olfactory and gustatory systems are admirable in themselves, and contain the results of much recent work not readily accessible in textbooks, but it is a little difficult to see why either of these special sensory systems should be classified as autonomic and parasympathetic. The argument given on page 147 in favour of this usage seems particularly weak and could be used to justify the inclusion of almost any afferent nerve in the autonomic system. There would seem to be even less justification for the inclusion of some of the other subjects, such as descriptions and figures of the sulci and gyri of the cerebral cortex, the cortical distribution of cerebral arteries and a number of others, most of which are described in greater detail in the standard textbooks of anatomy.

The criticism that a book contains more than one would expect to find, is not perhaps very serious. In the autonomic nervous system there are many features which are incompletely understood and much of a controversial nature, and there is room for disagreement over what should be included in it. While many will not agree with all the views expressed by Professor Mitchell, this book provides a comprehensive account of the anatomy of the innervation of viscera both efferent and afferent, considered from a broad but almost exclusively anatomical point of view. As such it is a valuable addition to medical literature; its controversial aspects will stimulate interest and, it may be hoped, further work, not only from anatomists but from physiologists and clinicians as well. It can be only from the co-operation of all three that the many problems of the autonomic nervous system will eventually be solved.

F.G.

\section{DISEASES OF THE CHEST}

Vols. I and II

Edited by Sir Geoffrey Marshall, K.C.V.O., C.B.E., M.D., F.R.C.P., and KENNETH M. A. Perry, M.A., M.D., F.R.C.P. Pp. xi +475 , with ${ }_{5} 8$ illustrations, and pp. vii +444 , with I 92 illustrations, respectively. London: Butterworth \& Co., Ltd. 1952. $\& 7$ 7s. the set.

Thirty-one experts have contributed to this textbook in two volumes which covers the whole field of chest diseases including tuberculosis. This is a formidable undertaking since books of equal length have been written on pulmonary tuberculosis alone.

It is claimed by the publishers that an even balance has been maintained between the sections concerning tuberculosis and other diseases; 208 pages have been devoted to the former and 657 to the latter. It is difficult to decide the relative importance of these two groups which may well change rapidly in the near future, but many chest specialists would probably suggest a more cven distribution. The tuberculosis section, although good, appears rather cramped and concerning treatment there is no mention of some important practical points, such as when to abandon an artificial pneumothorax especially in the presence of pleural thickening. The absence of this complication in pneumoperitoneum treatment is surely a great advantage. The period of maintenance of pneumoperitoneum recommended is two to three years - a rather short span for a less efficient form o collapse than artificial pneumothorax for which thẹ usual recommendation is five years.

The variability of the styles of the writers is striking, but is not distracting in the non-tuberculous sections where change of style usually coincides with change of disease. In the tuberculous section, however, these changes are rather disturbing to continuity of thought and may be confusing to the reader inexperienced in pulmonary tuberculosis.

In a book of this standard it is difficult to pick out sections for special praise and every critical reader would no doubt select a different group. The chapters dealing with bronchopulmonary anatomy, emphysema, applied physiology, haematogenous and pleural tuberculosis, pulmonary embolism and lung tumours must rank high on any list, and that dealing with asthma, a notoriously complex and 'partisan' disease, is a wise and balanced review.

The book is first class and fills a long-felt gap in medical literature. It is authoritative, full, practical and as up to date as possible. It is written almost entirely from personal experience and owes little or nothing to previous textbooks. As a whole, it is suitable as a reference book for chest specialists and it is therefore suggested that in future editions more space should be devoted to pulmonary tuberculosis, especially regarding treatment. Possibly this subject would be better treated by two authors - a physician and a surgeon.

The book is strongly recommended to membership candidates, general physicians and chest 
specialists. Its practical and common-sense approach makes it of interest and considerable value to general practitioners and although rather advanced for senior students, many would find it a stimulating and refreshing contrast to some standard textbooks.

\section{A TEXTBOOK OF CLINICAL NEUROLOGY}

By J. M. Nielsen, B.S., M.D., F.A.C.P. 3rd Edition. Pp. xiv +709 , with 212 illustrations. London: Cassell \& Co. 1951. £3 r5s. od.

This is a most readable book, practical, honest, clear, printed beautifully and well illustrated.

It has the great virtue of not being in the least alarming - a rare quality in textbooks of neurology.

It is not, however, a book of reference. Only larger and (in all senses) heavier books can serve this purpose. But it is an excellent book for anyone wishing to be introduced to a neurological subject or disease. Moreover it is really up to date - so much so that examinees would be well advised not to quote some of the treatments advised (such as intravenous procaine for cerebral thrombosis) which may be of great value but are as yet probably untried and certainly unaccepted in this country.

The chapters on recent advances (for example those on electroencephalography and angiography) are for the most part sound introductions to their subjects. They make no attempt to be comprehensive and so they avoid the dull, cataloguing atmosphere which makes so many books difficult to read.

A strange exception is the chapter on electromyography, from which it is difficult to get any clear view of the subject. The confusion is worst, oddly enough, in the plates to this chapter. It is almost impossible to follow their subtitles.

There are not a few statements and some classifications which are open to objection. What he calls progressive ophthalmoplegia of nuclear origin has a distribution indicating that it is far more probably a myopathy, and his claims for the use of tocopherol in amyotrophic lateral sclerosis would hardly be accepted here. Such objections, however, are inevitable in any live textbook on a live subject and they are relatively of minor importance.

This is outstandingly the type of book that a busy practitioner would enjoy. It is easy to dip into and quite difficult to put down.

It is certainly costly but the paper is luxurious, the lay-out spacious and the plates plentiful and good.

\section{C.E.}

\section{CARDIOSCOPY}

By William Evans, M.D., D.Sc., F.R.C.P. Pp. $\mathrm{xi}+$ 150, with 207 illustrations. London: Butterworth \& Co. r952. 40s.

It is a salutary experience for a radiologist to review a book written by a physician on a subject which is primarily of a radiological flavour.

To the purist the title 'Cardioscopy' might be criticized as misleading and the use of the terms ' outer border' and 'mesial heart shadow' in a description of the oblique views of the heart could lead to confusion; ' right and left borders as seen by the observer' would have been a happier choice of terminology.

These minor criticisms apart, this is an excellent little book or atlas of a wide range of cardiac conditions likely to be met with on fluoroscopic examination of the heart from displacement of the heart in chest deformities to behaviour of the heart in endocrine disfunctions.

Dr. Evans is to be congratulated on his choice of illustrations which are exceedingly well reproduced and really illustrate the points in the descriptive text, which is written in a straightforward manner and leaves no room for ambiguity, particularly on a subject which is difficult to transcribe from motion as seen to static cold print. On few occasions only does he transgress and confuse the fluorescent and the photographic image.

A medical student's curriculum is so packed one hesitates to load him with more and more reading, particularly of a specialized nature. There is, however, a hard core whose appetite for literature is insatiable, they will read and enjoy this book and the rest will benefit if only to look at the illustrations and read their captions. It is to the postgraduate student in diagnostic radiology and his colleague working for higher examinations in medicine that this book will most appeal. To the former, it forms a basis for the understanding of more elaborate and detailed reading in his radiological studies of the heart and to the latter a fund of practical knowledge which may tip the scales for a gold medal or that final viva voca examination.

T.R.R.

\section{PHYSICAL MEDICINE SERVICES Vol. I}

Muscle Relaxation as an Aid to Psychotherapy By G. Garmany, B.Sc., M.B., Ch.B., M.R.C.P., D.P.M. Pp. 65. London: The Actinic Press, Ltd. 1952. 3s. 6d.

This monograph explains the application of physical medicine to psychiatric treatment. The training of patients in muscle relaxation is part of the curriculum in the course of training of physiotherapists and the special application of this form of treatment is explained and described. The author rightly stresses the limitations of the treatment and the fact that it is only a part of the treatment which is prescribed by the psychiatrist in carefully selected cases.

It is a most readable and informative book for physiotherapists and medical men. 